\title{
Methodology for Performance Evaluation of Pre-departure Sequencing Tools in Terms of A-CDM Concept
}

\section{Hynek Hrabik ${ }^{1}$, Matej Nesvadba ${ }^{2}$, Stanislav Pleninger ${ }^{1 *}$}

\footnotetext{
${ }^{1}$ Department of Air Transport, Faculty of Transportation Sciences, Czech Technical University in Prague, Prague, Czech Republic

${ }^{2}$ Development and Planning Division, Air Navigation Services of the Czech Republic, Jenec u Prahy, Czech Republic

*Corresponding author: Czech Technical University in Prague, Faculty of Transportation Sciences, Department of Air Transport, Horská 3, 12803 Prague, Czech Republic, Email: pleninger@fd.cvut.cz
}

\begin{abstract}
The article describes the proposed methodology for performance evaluation of Pre-Departure Sequencing tools. Pre-departure management delivers optimal traffic flow to the runway by route planning and accurate taxi time forecasts. Firstly, a Pre-Departure Sequencer Start-Up Manager (SUM) used by Air Navigation Services of the Czech Republic (ANS CZ) at Vaclav Havel Airport Prague (LKPR) is mentioned. The main parts deal with the proposed methodology for performance evaluation of Start-Up Manager. The methodology uses several indicators to evaluate the performance. The methodology utilizes time milestones introduced in Airport Collaborative Decision Making (A-CDM) concept. It focuses especially on aircraft pre-departure sequencing processes. Methodology was tested on the Start-Up Manager used by Air Navigation Services (ANS) of the Czech Republic at Vaclav Havel Airport Prague (LKPR). Achieved performance results and its credibility in accordance with the proposed methodology are presented and discussed in the final part of this paper.

\section{Keywords}

Collaborative decision making - Departure sequeincing - Pre-departure sequencing - Start-Up manager Target start-up time - Target take-off time
\end{abstract}

\section{Introduction}

Poor operational predictability in comparison to en-route phase is the main problem of airports [1]. There are several factors that play a critical role in the time which aircraft spend at the airport. Improving predictability at aerodromes has been a task for air traffic management provided in Europe by Network Manager Operation Center (NMOC). Without going into further details the main goal of NMOC is a smooth flow of air traffic, delay reduction, managing the capacity of European airspace and cost reduction.

To tackle this issue Airport Collaborative Decision Making (A-CDM) concept has been introduced. The concept aims 
at reducing delays at airside and improving the predictability. Furthermore, the concept provides Air Traffic Controllers (ATCOs) with high quality data, based on which ATCOs can make better decisions to ensure continuous traffic flow at the airport.

However, considering more data when making a decision may increase the workload of a controller, therefore it is desirable to use software tools for processing A-CDM data and calculating outputs. These tools such as Departure Manager (DMAN), Arrival Manager (AMAN), and Pre-Departure Sequencer (PDS) can assess current situation from several perspectives and they are capable of finding more effective solutions [2].

\section{SUM used by ANS CZ}

As mentioned earlier, Start-Up Manager (SUM) is used to determine pre-departure sequence at Vaclav Havel Airport Prague (LKPR). The sequence is effectively determined in real-time according to current traffic situation at the airport.

ATC determines the optimum time of start-up or pushback, i.e. Target Start-Up Approval Time (TSAT) using StartUp Manager software tool so that the aircraft taxies to the RWY holding point continuously and takes-off with a minimal delay. TSAT value may be changed depending on the current traffic situation [3].

Even though a reduction of holding time for each aircraft is the main goal, simulations with the DLR/Eurocontrol DMAN have affirmed that a very strict approach is not feasible: a queue of two or three departures at the runway holding point is considered satisfactory [4].

SUM uses Target Take-Off Time (TTOT) to derive TSAT. However, calculations of TTOT does not consider various constraints such as wake vortex separation, speed category separation, etc. Instead of it, SUM uses a parameter Departure Capacity Update (DCU) to ensure time separation between aircraft.

\section{The methodology for performance evaluation of SUM}

Performance of SUM is captured by 5 indicators. Each indicator focuses on performance evaluation from the specific perspective. The indicators are as follows:

1. Fulfillment of planned departure (DEP) sequence

2. $\Delta \mathrm{TSAT}_{\min }$

3. Development of $\triangle \mathrm{TTOT}, \Delta \mathrm{TTOT}_{(A S A T)}$

4. $\triangle$ ASAT (Actual Start up Time)

\subsection{Fulfillment of planned DEP sequence}

This indicator shows whether the planned departure sequence corresponds to real departure sequence. Since SUM is PreDeparture Sequencer the indicator assesses the real number of departures versus the planned number of departures in a floating hour ${ }^{1}$ rather than specific order of departures.

Number of planned departures is defined by TTOTs, number of real departures is defined by Actual Target Take-Off Time (ATOT). TTOT of a specific flight may be recalculated during a Turn-Around process as result of new inputs processed by SUM. Therefore last calculated TTOT prior to Actual Start-Up Time (ASAT) is considered.

Sum of planned departure in each 5-minute intervals in a given day are denoted as $s_{1}, s_{2}, s_{3}, \ldots, s_{288}$. Number of departures $P_{h}$ based on TTOT $(A S A T)$ in a given hour can be determined as:

$$
P_{h}=\sum_{i=m}^{n} s_{i},
$$

where $m$ denotes order of the first interval of a given hour in a day, $n$ denotes order of the last interval of a given hour in a day and index $h$ denote order of hour. Number of departures in the first floating hour based on TTOT $_{(A S A T)}$ can be determined as:

$$
P_{1}=\sum_{i=1}^{12} n_{i}
$$

Number of departure based on ATOT is determined analogously to the previous case. Variables $k_{1}, k_{2}, k_{3}, \ldots, k_{288}$ denote the sum of real departures in each 5-minute interval in a given day. Number of departures $\mathrm{P}^{\prime}$ in the first floating hour based on ATOT can be determined as:

$$
P_{1}^{\prime}=\sum_{i=1}^{12} k_{i} .
$$

\section{$3.2 \Delta$ TSATmin}

Indicator $\Delta \mathrm{TSAT}_{\text {min }}$ expresses time which $\mathrm{SUM}$ sets to each aircraft so that the aircraft taxies to RWY holding point continuously and takes-off with minimal delay. In other words it is a difference between the last calculated TSAT prior to Star-Up and minimal possible TSAT min $_{\text {. }}$

In general, the indicator $\Delta \mathrm{TSAT}_{\min }$ is determined as:

$$
\Delta T S A T_{\min }=T S A T-T S A T_{\min },
$$

where TSAT $_{\text {min }}$ is minimal possible time for issuing Start-Up Clearance, TSAT is last calculated TSAT prior to Start-Up. $\mathrm{TSAT}_{\text {min }}$ for regulated flight is defined as

$$
\Delta T S A T_{\min }=T S A T-M A X\{C T O T-E X O T, T O B T\},
$$

and $\mathrm{TSAT}_{\min }$ for non-regulated flight is defined as

$$
\Delta T S A T_{\text {min }}=T S A T-M A X\{T O B T, E O B T-10 M I N\},
$$

\footnotetext{
${ }^{1}$ Floating hour consist of 12 time intervals. Each interval represents a 5-minute time period of a day. For example a floating hour is from 1200 until 1300. The following floating hour is from 1205 until 1305 etc
} 
where CTOT is calculated off-block time, EXOT is estimated taxi-out time, TOBT is target off-block time and EOBT is estimated off-block time.

\section{3 $\triangle$ TTOT $_{(A S A T)}$, $\triangle$ TTOT development}

TTOT for a given flight might be recalculated several times since the first TTOT calculation. The first calculation is done 46 minutes prior to Estimated Off-Block Time (EOBT) of the flight as long as Target Off-Block Time (TOBT) has been set. The recalculation is done when Calculated Off-Block Time (CTOT), EOBT or TOBT of a flight is updated.

The last TTOT update is based on Actual Off-Block Time (AOBT). Finally, Actual Target Take-Off Time (ATOT) is obtained from Advanced Surface Movement Guidance and Control System (A-SMGCS) or it can be inserted by ATCO. Indicator $\triangle \mathrm{TTOT}$ evaluates development of the deviation defined as:

$$
\triangle T T O T=A T O T-T T O T_{n-i} .
$$

The value of index $n$ is equal to the number of TTOT recalculation of a given flight. The value may vary depending on a flight. The value of index $i$ goes from 0 to $n-1$. These two indexes define order of TTOT recalculation of a given flight.

$\triangle \mathrm{TTOT}_{(A S A T)}$ indicates difference between the real and the planned sequence. Indicator $\triangle \mathrm{TTOT}_{(A S A T)}$ is defined as:

$$
\triangle T T O T_{A S A T}=A T O T-T T O T_{A S A T} .
$$

\section{$3.4 \triangle$ ASAT}

Each stakeholder at LKPR must meet their requirements so that SUM works effectively. A-CDM procedures need to be followed by the crew, Ground Handling Agent (GHA) and Clearance Delivery. According to the procedures crew must request Start-Up Clearance within time window TSAT \pm 3 min.

The main reason why this indicator has been proposed is following. SUM states planned sequence and the actual sequence is executed by ATCO. However, if a planned situation does not correspond with a real one it might be impossible for ATCO to follow the procedures. For example because of excessive holding the ATCO would not issue the clearance within the time window to re-establish continuous flow of taxing aircraft. So the indicator $\triangle \mathrm{ASAT}$ demonstrates whether A-CDM procedure can be followed $[5,6]$. Indicator $\triangle A S A T$ is defined as:

$$
\triangle A S A T=A S A T-T S A T_{A S A T},
$$

where $\operatorname{TSAT}_{(A S A T)}$ is the last calculated TSAT prior to issuing Start-Up Clearance by ATCO.

\section{Selected results of the Analysis}

The results of the analysis using proposed methodology are discussed in this chapter. Data used in the analysis were provided by ANS of the Czech Republic and cover time period from 2015/06/01 until 2015/06/30. Number of processed data may slightly vary depending on the indicator nevertheless, 162 departures were analyzed a day in average. In total data of 4618 departures using A-CDM procedures were processed. Since the analyzed time period was in June, there were no Low Visibility Procedures (LPV) applied, which could have resulted in the airport capacity reduction.

The departure flow at LKPR in the average day ${ }^{2}$ is plotted on Fig. 1. There are 2 operational peaks, one of them from 10:05 until 11:05 with 16 departures, the second one from 15:35 until 15:35 until 16:35 with 12 departures. Particularly in these peak hours SUM is supposed to maintain continuous traffic and prevent from excessive holding prior to departures. In theory if parameter Departure Capacity Update (DCU) is set to 1.5 minutes, departure capacity is 40 departures in one hour without arrival flow and other delays.

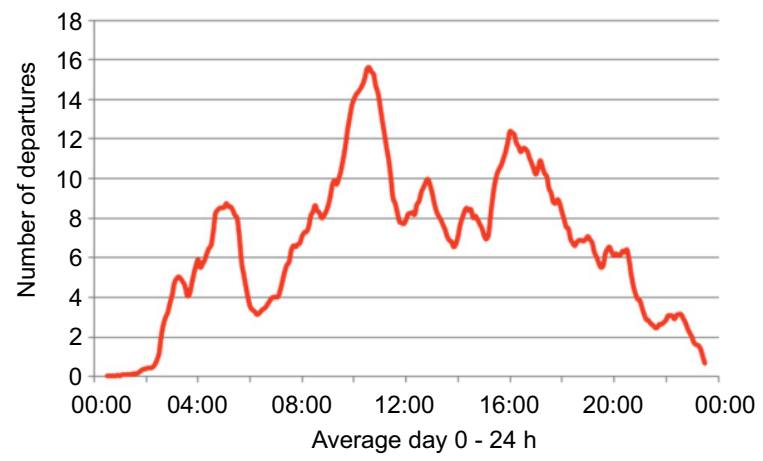

Figure 1. Departure flow at LKPR in average day.

\subsection{Fulfilment of planned DEP sequence}

The results of the analysis using this indicator are shown on Fig. 2. The planned sequence was fulfilled in interval $(95 ; 105) \%$ in $59 \%$ of flowing hours. More than $100 \%$ fulfilment of the sequence may be a result of previous less than $100 \%$ fulfilment.

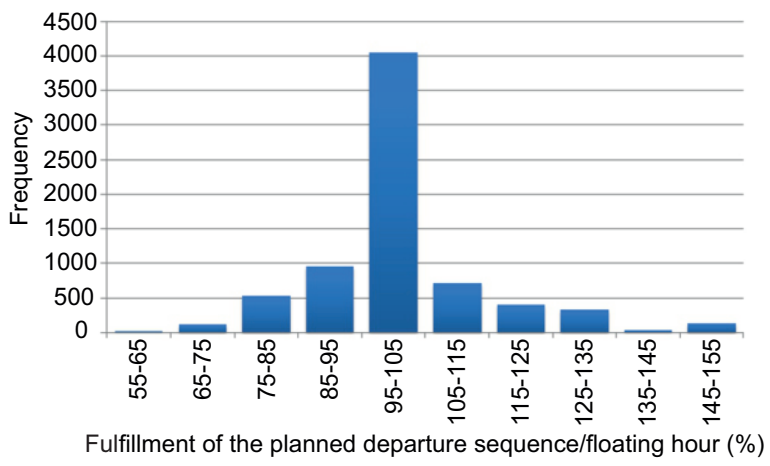

Figure 2. Histogram of fulfilment of the planned departure sequence.

\footnotetext{
${ }^{2}$ The average day consist of the average floating hours. Value of the indicator or the number of departures in the average floating hour is equal to average number of departures for the whole time period in the given floating hour.
} 


\section{2 $\Delta$ TSAT $_{\min }$}

As can be seen on Fig. 3, 47\% departures (2042) had $\Delta$ TSAT $_{\text {min }}$ $\leq 1 \mathrm{~min}$. The exponential distribution can be expected according to analysed data. Frequency increases if $\Delta \mathrm{TSAT}_{\text {min }}$ is within time interval 0:10:00-0:11:00. The increase was caused by penalization of the flights that did not request START-UP Clearance within time interval TSAT \pm 3 min.

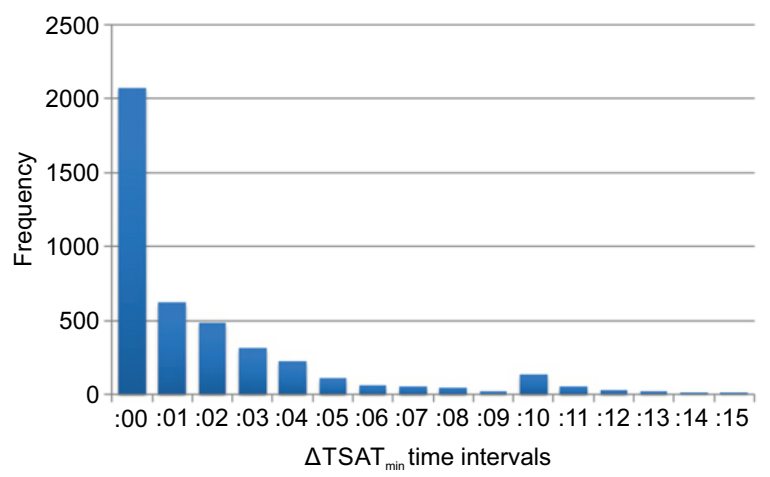

Figure 3. Histogram of $\Delta \mathrm{TSAT}_{\text {min }}$

Figure 4 displays causal relationship between the $\Delta \mathrm{TSAT}_{\min }$ average value of a departure and number of departures per hour. As expected SUM calculates greater value of $\Delta$ TSAT $_{\text {min }}$ for each flight during peak hours in order to maintain continuous departure flow.

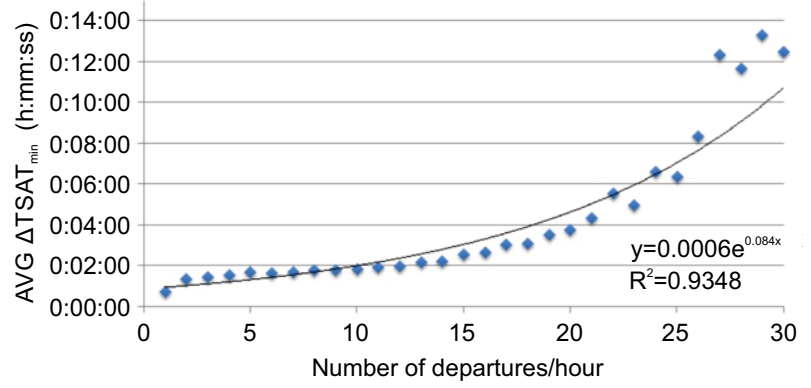

Figure 4. Average $\Delta \mathrm{TSAT}_{\min } \mathrm{vs}$. number of departures

\section{3 $\Delta$ TTOT $_{(A S A T)}$, $\triangle$ TTOT Development}

According to processed data $67 \%$ of the departures had ATOT later than $\triangle$ TTOT $_{(A S A T)}$. Table 1 summarize the analyzed data. The data are grouped by their value into time intervals. The intervals are in the left column, percentage of flights whose $\triangle \mathrm{TTOT}_{(A S A T)}$ are within the interval can be found in the right column.

Figure 5 shows positive results of the analysis. Even though there are several peak hours designed by the red curve during the average day, $\triangle$ TTOT $_{(A S A T)}$ marked by the blue curve does not correspond to the number of departures, which means that continuous flow of departures is sustained.

Finally, the Fig. 6 confirms, that in average every TTOT recalculation is more precise than its previous estimated value. The data plotted in the chart show no correlation between precision of the last calculated TTOT and number of recalculations.

Table 1. $\mid \triangle$ TTOT $_{(A S A T)} \mid$ time intervals

\begin{tabular}{cc}
$\left|\Delta \mathbf{T T O T}_{(A S A T)}\right|$ & DEP $(\%)$ \\
\hline$\langle 0-1\rangle \min$ & 13 \\
\hline$\langle 0-2\rangle \min$ & 33 \\
\hline$\langle 0-3\rangle \min$ & 48 \\
\hline$\langle 0-4\rangle \min$ & 62 \\
\hline$\langle 0-5\rangle \min$ & 72 \\
\hline$\langle 0-6\rangle \min$ & 80 \\
\hline$\langle 0-7\rangle \min$ & 85 \\
\hline$\langle 0-8\rangle \min$ & 89 \\
\hline$\langle 0-9\rangle \min$ & 92 \\
\hline$\langle 0-10\rangle \min$ & 94 \\
\hline \multicolumn{2}{c}{}
\end{tabular}

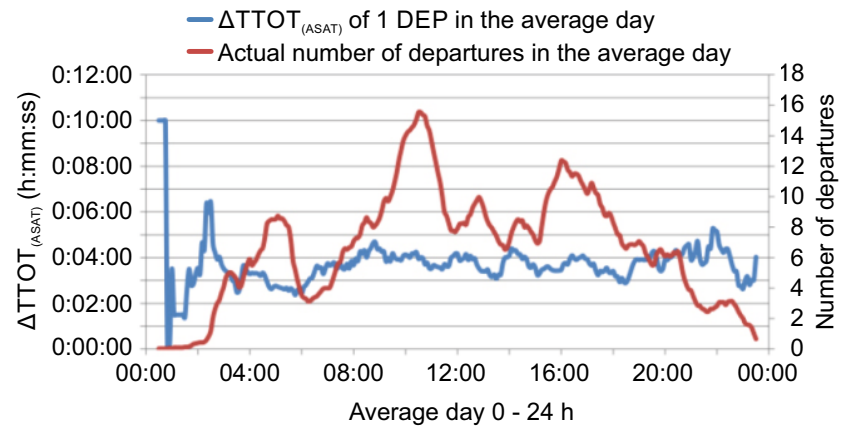

Figure 5. $\triangle$ TTOT $_{(A S A T)}$

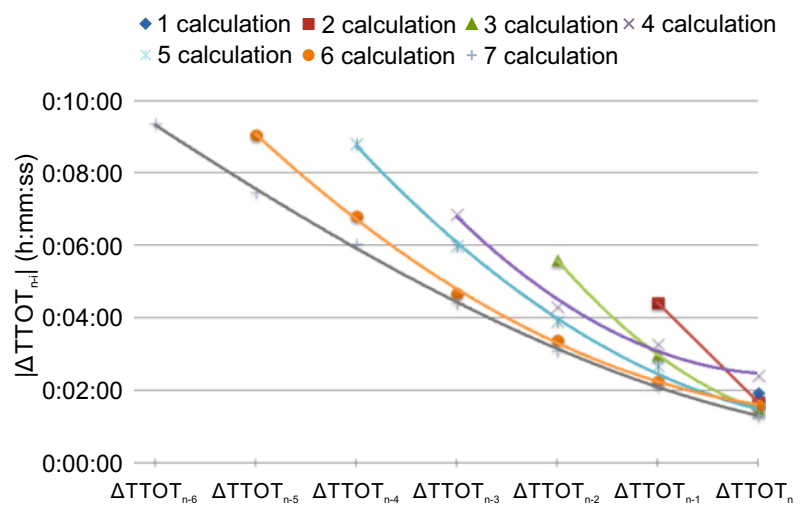

Figure 6. Development of $\left|\Delta \mathrm{TTOT}_{(A S A T)}\right|$

\section{Conclusion}

This article discusses proposed methodology for performance evaluation pre-departure sequencing tools. The methodology is based on uses several indicators to evaluate the performance. The following indicator were proposed: Fulfilment of planned departure sequence, Difference between the last calculated TSAT prior to Star-Up and minimal possible $\mathrm{TSAT}_{\text {min }}\left(\Delta \mathrm{TSAT}_{\text {min }}\right)$, Development of delta Target Take-Off 
Time $\left(\triangle \operatorname{TTOT}_{(A S A T)}\right)$, Difference between ASAT and last calculated TSAT prior to issuing Start-Up Clearance by ATCO $(\triangle \mathrm{ASAT})$.

Methodology was tested on sample of operational data at Vaclav Havel Airport Prague (LKPR) in June 2015 where Start-Up Manager (SUM) was used. Achieved results are presented.

The methodology may be utilized to analyze real A-CDM data and the results of the analysis may help to decide what steps need to be taken to optimize procedures when using the tools. How to optimize the tool or to compare the performance of two or more similar products [7].

\section{References}

[1] S. Niarchakou and J. Simón Selva. ATFCM Operations Manual: Network Operations Handbook, 2016. URL http://www.eurocontrol.int/ sites/default/files/content/documents/ $\mathrm{nm} /$ network-operations/HANDBOOK/ ATFCM-Operations-Manual-current.pdf. [Online].

[2] Eugene Tuinstra and Klaus Haschke. Generic Operational Concept for Pre-departure Runway Sequence Planning and Accurate Take-Off Performance: Enabled by DMAN interaction with Airport CDM and A-SMGCS concepts. Version 07A, 2009. URL http : / / www . yumpu . com/ en/document/view/19559173/. [Online].

[3] Air Navigation Services of the Czech Republic. LKPR AD 2.20 Local Traffic Regulations, 2016. URL https://lis.rlp.cz/ais_data/aip/data/ valid/a2-pr-txt2.pdf. [Online].

[4] Meilin Schaper, Gina Tsoukala, Rania Stavrati, and Nikos Papadopoulos. Departure flow control through takeoff sequence optimisation: Setup and results of trials at athens airport. In 2011 IEEE/AIAA 30th Digital Avionics Systems Conference. Institute of Electrical and Electronics Engineers (IEEE), oct 2011. doi: 10.1109/dasc. 2011.6095986. URL https: / / doi .org/10 . 1109\% 2Fdasc.2011.6095986.

[5] EUROCONTROL. Airport CDM Implementation Manual Version 4, 2012. URL http: / / www . eurocontrol. int/download/publication/node-field_ download-4929-0. [Online].

[6] Jakub Kraus. Determining acceptable level of safety of approach to landing. In Proceedings of 20th International Conference Transport Means 2016, pages 230-235. Kauno technologijos universitetas (KTU), oct 2016.

[7] Ioannis Simaiakis, Harshad Khadilkar, Hamsa Balakrishnan, Tom G. Reynolds, and R. John Hansman. Demonstration of reduced airport congestion through pushback rate control. Transportation Research Part A: Policy and
Practice, 66:251-267, aug 2014. doi: 10.1016/j.tra.2014. 05.014. URL https://doi.org/10.1016\%2Fj. tra.2014.05.014. 